PROCEEDINGS OF THE

AMERICAN MATHEMATICAL SOCIETY

Volume 139, Number 5, May 2011, Pages 1645-1658

S 0002-9939(2010)10594-4

Article electronically published on September 3, 2010

\title{
A RANGE AND EXISTENCE THEOREM FOR PSEUDOMONOTONE PERTURBATIONS OF MAXIMAL MONOTONE OPERATORS
}

\author{
VY KHOI LE
}

(Communicated by Nigel J. Kalton)

\begin{abstract}
In this paper, we prove a range and existence theorem for multivalued pseudomonotone perturbations of maximal monotone operators. We assume a general coercivity condition on the sum of a maximal monotone and a pseudomonotone operator instead of a condition on the pseudomonotone operator only. An illustrative example of a variational inequality in a Sobolev space with variable exponent is given.
\end{abstract}

\section{INTRODUCTION}

We are concerned in this paper with a range and existence theorem for multivalued pseudomonotone perturbations of maximal monotone operators and its corollaries. In the theorem, as in Theorem 2.1, 7] and Theorem 6.1, 8], we assume a coercivity condition on the sum of a maximal monotone and a pseudomonotone operator rather than on the pseudomonotone operator solely (cf. e.g. 1, 4, 3, 9, 10, 13, 14]). As consequences, we obtain improvements and unifications over a number of theorems in which various types of conditions were assumed, for example, in Theorem 5.2 and Corollary 5.2 of [10, where the coercivity condition was assumed on the pseudomonotone operator only or a linear growth (from below) of the pseudomonotone operator. We also obtain as corollaries existence theorems for variational inequalities containing multivalued pseudomonotone operators. As an illustrating example for the abstract results, we study the existence of solutions to a variational inequality in a Sobolev space with variable exponent.

\section{MAIN THEOREM}

Let $X$ be a reflexive Banach space with norm $\|\cdot\|$, dual $X^{*}$, dual norm $\|\cdot\|_{*}$, and dual pairing $\langle\cdot, \cdot\rangle$. We shall use " - " for the weak convergence in $X$ and " ${ }^{*}$ " for the weak* convergence in $X^{*}$. This different notation is only for clarity and is not essential since the weak and weak* convergences are the same in $X^{*}$. Assume $A, B: X \rightarrow 2^{X^{*}}$ are multivalued operators from their corresponding domains $D(A)$ and $D(B)$ into the subsets of $X^{*}$. As usual, $R(A)$ denotes the range of $A$. We shall use the definitions of maximal monotone and multivalued pseudomonotone

Received by the editors March 9, 2010 and, in revised form, May 10, 2010.

2010 Mathematics Subject Classification. Primary 35J85, 47H05, 47J20, 47J30.

(C)2010 American Mathematical Society 
operators together with their properties as presented in [4, 10, 13], etc. Their definitions are included here to avoid confusion.

Definition 2.1. (a) A mapping $A: X \rightarrow 2^{X^{*}}$ is called monotone if

$$
\left\langle u^{*}-v^{*}, u-v\right\rangle \geq 0, \text { for all }\left(u, u^{*}\right),\left(v, v^{*}\right) \in \operatorname{Gr}(A),
$$

where $\operatorname{Gr}(A):=\left\{\left(w, w^{*}\right) \in X \times X^{*}: w^{*} \in A(w)\right\}$ is the graph of $A$.

A monotone mapping $A$ is called maximal monotone if the graph of $A$ is a maximal subset of $X \times X^{*}$ with respect to the set inclusion partial ordering of $X \times X^{*}$.

(b) A mapping $B: X \rightarrow 2^{X^{*}}$ is called pseudomonotone if

(i) $B(u)$ is closed, bounded, and convex for all $u \in D(B)$;

(ii) $B$ is upper semicontinuous from each finite-dimensional subspace of $X$ to $X^{*}$ with the weak topology;

(iii) $\left\{u_{n}\right\}$ is a sequence in $X$ and $\left\{u_{n}^{*}\right\}$ a sequence in $X^{*}$ such that $u_{n} \rightarrow u$, $u_{n}^{*} \in B\left(u_{n}\right), \forall n \in \mathbb{N}$, and $\limsup _{n \rightarrow \infty}\left\langle u_{n}^{*}, u_{n}-u\right\rangle \leq 0$, then for each $v \in X$ there exists $u^{*}(v) \in B(u)$ such that $\liminf _{n \rightarrow \infty}\left\langle u_{n}^{*}, u_{n}-v\right\rangle \geq\left\langle u^{*}(v), u-v\right\rangle$.

In the sequel, we use the notation $B_{R}(0)=\{u \in X:\|u\|<R\}$ for the open ball centered at 0 with radius $R, \overline{B_{R}(0)}=\{u \in X:\|u\| \leq R\}$ for the closed ball centered at 0 with radius $R$, and $S_{R}(0)=\{u \in X:\|u\|=R\}$ for the corresponding sphere.

Our main result is the following theorem.

Theorem 2.2. Let $A: D(A)(\subset X) \rightarrow 2^{X^{*}}$ be a maximal monotone operator, $B: D(B)=X \rightarrow 2^{X^{*}}$ be a bounded multivalued pseudomonotone operator, and $L \in X^{*}$. Assume there exists $u_{0} \in X$ and $R \geq\left\|u_{0}\right\|$ such that $D(A) \cap B_{R}(0) \neq \emptyset$ and

$$
\left\langle\xi+\eta-L, u-u_{0}\right\rangle>0,
$$

for all $u \in D(A)$ with $\|u\|=R$, all $\xi \in A(u), \eta \in B(u)$. Then the inclusion

$$
A(u)+B(u) \ni L
$$

has a solution, i.e., $L \in R(A+B)$.

Proof. The proof is divided into several steps.

Step 1 . We prove that given any $u_{0} \in X$, there exists a continuous, strictly increasing function $f:[0, \infty) \rightarrow[0, \infty)$ such that $f(0)=0, \lim _{r \rightarrow \infty} f(r)=+\infty$, and for some $M \geq 0$

$$
\inf _{\eta \in B(u)}\left\langle\eta, u-u_{0}\right\rangle \geq-f(\|u\|)-M, \forall u \in X .
$$

In fact, for $r \geq 0$, put $f_{1}(r)=\sup \left\{\|\eta\|_{*}: \eta \in B(u),\|u\| \leq r\right\}$. By the boundedness of $B, f_{1}(r) \in[0, \infty), \forall r \in[0, \infty)$. Moreover, from its definition $f_{1}$ is increasing on $[0, \infty)$ and

$$
\|\eta\|_{*} \leq f_{1}(\|u\|), \forall u \in X, \eta \in B(u) .
$$

Let $f_{2}$ be a continuous, strictly increasing function from $[0, \infty)$ to $[0, \infty)$ such that

$$
f_{1}(r) \leq f_{2}(r), \forall r \in[0, \infty),
$$


and $\lim _{r \rightarrow \infty} f_{2}(r)=\infty$. The function $f_{2}$ can be simply constructed as a piecewise linear function as follows. Let $a_{0}=1+\sup \left\{f_{1}(r): r \in[0,1]\right\}$ and

$$
a_{n}=1+\max \left\{a_{n-1}, \sup \left\{f_{1}(r): r \in[n, n+1]\right\}\right\} \quad \text { for } n=1,2, \ldots
$$

We define $f_{2}$ by $f_{2}(t)=a_{n}$ if $t \in\{0,1,2, \ldots\}$ and $f_{2}(t)$ is linear in the interval $[n, n+1]$ for all $n \in\{0,1,2, \ldots\}$. It is clear from this construction that $f_{2}(r) \rightarrow \infty$ as $r \rightarrow \infty$.

For $u \in X$ with $\|u\| \leq\left\|u_{0}\right\|$ and for any $\eta \in B(u)$, we have from (2.4) and (2.5) that

$$
\left|\left\langle\eta, u-u_{0}\right\rangle\right| \leq\|\eta\|_{*}\left(\|u\|+\left\|u_{0}\right\|\right) \leq f_{1}(\|u\|)\left(2\left\|u_{0}\right\|\right) \leq 2 f_{2}\left(\left\|u_{0}\right\|\right)\left\|u_{0}\right\| .
$$

For $u \in X$ with $\|u\| \geq\left\|u_{0}\right\|$ and for $\eta \in B(u)$, as in the above estimate, we have

$$
\left|\left\langle\eta, u-u_{0}\right\rangle\right| \leq 2 f_{2}(\|u\|)\|u\| \text {. }
$$

Hence, for all $u \in X$, all $\eta \in B(u)$,

$$
\left|\left\langle\eta, u-u_{0}\right\rangle\right| \leq f(\|u\|)+M
$$

with $M=2 f_{2}\left(\left\|u_{0}\right\|\right)\left\|u_{0}\right\| \geq 0$ and $f(r)=2 r f_{2}(r)(r \geq 0)$. It is clear that $f$ is continuous and strictly increasing from $[0, \infty)$ into itself, $f(0)=0$, and $f(r) \rightarrow \infty$ as $r \rightarrow \infty$. Also, (2.3) is an immediate consequence of (2.8).

Step 2. Let $\alpha$ be a positive number (the choice of $\alpha$ is given in the next step). Let us define

$$
g(t)=2 \alpha t f(2 \alpha t), t \in[0, \infty),
$$

with $f$ given in Step 1. We see that $g(t)$ is also a continuous, strictly increasing function from $[0, \infty)$ into itself with $g(0)=0$ and $g(t) \rightarrow \infty$ as $t \rightarrow \infty$. We construct in this step a continuous, strictly increasing, convex function $\Phi$ from $[0, \infty)$ into itself such that $\Phi(0)=0$ and

$$
[\Phi(r)-\Phi(s)](r-s) \geq g(|r-s|)-M_{0}, \forall r, s \in[0, \infty),
$$

for some constant $M_{0}$ (independent of $r$ and $s$ ). We first define a sequence $\left\{b_{n}\right\}$ inductively as follows. Let $b_{0}=0, b_{1}=g(3)+b_{0}+1=g(3)+1$, and in general,

$$
b_{n+1}=1+\max \left\{2 b_{n}-b_{n-1}, \max \left\{b_{k}+g(n+3-k): k \in\{0,1, \ldots, n\}\right\}\right\} .
$$

We define the function $\Phi(t)(t \in[0, \infty))$ by $\Phi(n)=b_{n}$ for all $n \in\{0,1,2, \ldots\}$, and $\Phi(t)$ is linear on each interval $[n, n+1](n=0,1,2 \ldots)$. Since $0 \leq b_{n}<b_{n+1}$ and $b_{n+1}-b_{n}>b_{n}-b_{n-1}$ for all $n$, $\Phi$ is continuous, strictly increasing, and convex from $[0, \infty)$ into itself. Clearly $\Phi(0)=0$ and since $b_{n+1} \geq b_{n}+1, \forall n$, we see that $\Phi(t) \rightarrow \infty$ as $t \rightarrow \infty$.

Let $r, s \geq 0$ and assume without loss of generality that $r \geq s$. First, let us consider the case where $r-s \geq 2$. There are unique $n, m \in\{0,1,2, \ldots\}$ such that

$$
n \leq s<n+1 \text { and } m \leq r-s<m+1 .
$$

We have $m \geq 2$ in this case. It follows from (2.12) that $r \geq n+m$ and therefore

$$
\Phi(r) \geq \Phi(n+m) \text { and } \Phi(s)<\Phi(n+1) .
$$

Since $n+m \geq n+2$, we have from (2.11) that

$$
b_{n+m}>b_{k}+g(n+m+2-k), k \in\{0,1, \ldots, n+m-1\} .
$$


With $k=n+1 \leq n+m-1$, we have

$$
\Phi(n+m)=b_{n+m}>b_{n+1}+g(m+1)=\Phi(n+1)+g(m+1) .
$$

Hence, from (2.13), we obtain

$[\Phi(r)-\Phi(s)](r-s) \geq \Phi(r)-\Phi(s) \geq \Phi(n+m)-\Phi(n+1) \geq g(m+1) \geq g(r-s)$.

We have shown that

$$
[\Phi(r)-\Phi(s)](r-s) \geq g(|r-s|) \geq g(|r-s|)-g(2),
$$

for all $r, s \in[0, \infty)$ with $|r-s| \geq 2$. If $|r-s| \leq 2$, then $g(|r-s|) \leq g(2)$, and since $\Phi$ is increasing,

$$
[\Phi(r)-\Phi(s)](r-s) \geq 0 \geq g(|r-s|)-g(2) .
$$

From (2.14) and (2.15), we get (2.10) for all $r, s \in[0, \infty)$ with $M_{0}=g(2)(\geq 0)$.

Step 3. Since $X$ is reflexive, there exists a norm $\|\cdot\|_{0}$ on $X$ such that $X$ and $X^{*}$ are strictly convex under $\|\cdot\|_{0}$ and its corresponding dual norm $\|\cdot\|_{0 *}$. Let $\alpha \geq 1$ be such that

$$
\alpha^{-1}\|u\| \leq\|u\| \leq \alpha\|u\|, \forall u \in X,
$$

and let $\Phi$ be the function constructed in Step 2 with this $\alpha$. From Proposition 2.3, [12, there exists a (unique, single-valued) duality mapping $Q:\left(X,\|\cdot\|_{0}\right) \rightarrow\left(X^{*}, \| \cdot\right.$ $\left.\|_{0 *}\right)$ associated with $\Phi$, that is,

$$
\langle Q(u), u\rangle=\|Q(u)\|_{0 *}\|u\|_{0},
$$

and

$$
\|Q(u)\|_{0 *}=\Phi\left(\|u\|_{0}\right), \forall u \in X
$$

It is known (cf. Proposition 2.1, 12] ) that $Q$ is a monotone operator with $D(Q)=X$ and

$$
\langle Q(u)-Q(v), u-v\rangle \geq\left[\Phi\left(\|u\|_{0}\right)-\Phi\left(\|v\|_{0}\right)\right]\left(\|u\|_{0}-\|v\|_{0}\right), \forall u, v \in X .
$$

From (2.18), $Q$ is a bounded mapping; moreover, $Q$ is hemicontinuous on $X$. According to Proposition 2.5, [12, $Q$ is a (single-valued) bounded pseudomonotone mapping from $X$ to $X^{*}$ with $D(Q)=X$. Let $u_{0}$ be a fixed element in $D(A)$. Let $u, v \in X$. If $\|u\| \geq 2 \alpha^{2}\|v\|$, then from (2.19) and (2.9) $-(2.10),\|u\|_{0} \geq 2\|v\|_{0}$ and thus

$$
\begin{aligned}
\langle Q(u)-Q(v), u-v\rangle & \geq g\left(\|u\|_{0}-\|v\|_{0}\right)-M_{0} \\
& \geq 2 \alpha\left(\|u\|_{0}-\|v\|_{0}\right) f\left[2 \alpha\left(\|u\|_{0}-\|v\|_{0}\right)\right]-M_{0} .
\end{aligned}
$$

Since $\|u\|_{0}-\|v\|_{0} \geq \frac{1}{2}\|u\|_{0}$, by (2.16) and the increasing monotone property of $f$, we have the following estimate:

$$
\langle Q(u)-Q(v), u-v\rangle \geq \alpha\|u\|_{0} f\left(\alpha\|u\|_{0}\right)-M_{0} \geq\|u\| f(\|u\|)-M_{0}
$$

(if $\|u\| \geq 2 \alpha^{2}\|v\|$ ).

Step 4 . Let $L \in X^{*}$. Let $R$ be a positive number such that $D(A) \cap B_{R}(0) \neq \emptyset$ and let $I_{R}=I_{\overline{B_{R}(0)}}$ be the indicator functional of the closed ball $\overline{B_{R}(0)}$ :

$$
I_{R}(u)=\left\{\begin{array}{lll}
0 & \text { if } & u \in \overline{B_{R}(0)} \\
\infty & \text { if } & u \notin \overline{B_{R}(0)}
\end{array}\right.
$$


$I_{R}$ is a convex, lower semicontinuous, proper functional from $X$ to $[0, \infty]$ with $D\left(I_{R}\right)=\overline{B_{R}(0)}$. Its subdifferential $\partial I_{R}: D\left(\partial I_{R}\right) \rightarrow 2^{X^{*}}$ is a maximal operator with domain $D\left(\partial I_{R}\right)=D\left(I_{R}\right)=\overline{B_{R}(0)}$. Thus, $D(A) \cap\left[D\left(\partial I_{R}\right)\right]^{\circ}=D(A) \cap B_{R}(0) \neq$ $\emptyset$, which implies that $A+\partial I_{R}$ is a maximal monotone operator and moreover $D\left(A+\partial I_{R}\right)=D(A) \cap \overline{B_{R}(0)}$ is a bounded set.

Let $Q$ be constructed in Step 3. For $\varepsilon \in(0,1)$ (being fixed in this step), we consider the inclusion of finding

$$
u \in D\left(A+\partial I_{R}\right)\left(=D(A) \cap \overline{B_{R}(0)}\right)
$$

such that

$$
\left(A+\partial I_{R}\right)(u)+(B+\varepsilon Q)(u) \ni L,
$$

that is, finding $u$ satisfying (2.21) and $\zeta \in\left(A+\partial I_{R}\right)(u)$ and $\eta \in B(u)$ such that

$$
\zeta+\eta+\varepsilon Q(u)=L .
$$

Note that $B+\varepsilon Q$ is a (multivalued) bounded pseudomonotone operator with domain $D(B+\varepsilon Q)=X$. We prove that (2.23) (i.e. (2.22) ) has a solution. Let $u_{1}$ be any (fixed) element of $D(A) \cap \overline{B_{R}(0)}$. We check the following coercivity condition:

$$
\inf _{\mu \in(B+\varepsilon Q)(u)} \frac{\left\langle\mu, u-u_{1}\right\rangle}{\|u\|} \rightarrow \infty \text { as }\|u\| \rightarrow \infty .
$$

In fact, let $u \in X$ with $\|u\| \geq \max \left\{2 \alpha^{2}\left\|u_{1}\right\|, 2 \varepsilon^{-1}\right\}$ and $\xi \in B(u), \mu=\eta+\varepsilon Q(u) \in$ $(B+\varepsilon Q)(u)$. We have from (2.20) with $v=u_{1}$ that

$$
\left\langle Q(u)-Q\left(u_{1}\right), u-u_{1}\right\rangle \geq\|u\| f(\|u\|)-M_{0} .
$$

Hence,

$$
\left\langle Q(u), u-u_{1}\right\rangle \geq\|u\| f(\|u\|)-M_{0}-\left\|Q\left(u_{1}\right)\right\|_{*}\left(1+\frac{1}{2 \alpha^{2}}\right)\|u\| .
$$

It follows from (2.3) and this estimate that

$$
\begin{aligned}
\left\langle\mu, u-u_{1}\right\rangle & =\left\langle\eta, u-u_{1}\right\rangle+\varepsilon\left\langle Q(u), u-u_{1}\right\rangle \\
& \geq-f(\|u\|)-M+\varepsilon\|u\| f(\|u\|)-\varepsilon M_{0}-\varepsilon\left\|Q\left(u_{1}\right)\right\|_{*}\left(1+\frac{1}{2 \alpha^{2}}\right)\|u\| \\
& \geq(\varepsilon\|u\|-1) f(\|u\|)-M-M_{0}-\left\|Q\left(u_{1}\right)\right\|_{*}\left(1+\frac{1}{2 \alpha^{2}}\right)\|u\| \\
& \geq \frac{\varepsilon}{2}\|u\| f(\|u\|)-M-M_{0}-\left\|Q\left(u_{1}\right)\right\|_{*}\left(1+\frac{1}{2 \alpha^{2}}\right)\|u\| .
\end{aligned}
$$

Thus, for all $u \in X$ with $\|u\| \geq \max \left\{2 \alpha^{2}\left\|u_{1}\right\|, 2 \varepsilon^{-1}\right\}$, for all $\mu \in(B+\varepsilon Q)(u)$, we have

$$
\frac{\left\langle\mu, u-u_{1}\right\rangle}{\|u\|} \geq \frac{\varepsilon}{2} f(\|u\|)-\left\|Q\left(u_{1}\right)\right\|_{*}\left(1+\frac{1}{2 \alpha^{2}}\right)-\frac{M+M_{0}}{\|u\|} .
$$

Since $f(r) \rightarrow \infty$ as $r \rightarrow \infty$, this estimate immediately implies (2.24). We have verified that $A+\partial I_{R}$ and $B+\varepsilon Q$ satisfy the conditions of Theorem 5.2, [10, (see also similar results such as Theorem 3.2 in 9], Theorem 7 in [4, or Theorem 2.12 in [13). According to that theorem, (2.23) (or equivalently, (2.22) ) has at least one solution $u \in D\left(A+\partial I_{R}\right)$. 
Step 5. Let $R$ be as in Step 4. For each $\varepsilon \in(0,1)$, let $u=u_{\varepsilon} \in D\left(A+\partial I_{R}\right)$ be a solution of (2.23), that is, $u_{\varepsilon} \in D(A) \cap \overline{B_{R}(0)}$ and there are $\zeta_{\varepsilon} \in\left(A+\partial I_{R}\right)\left(u_{\varepsilon}\right)$ and $\eta_{\varepsilon} \in B\left(u_{\varepsilon}\right)$ such that

$$
\zeta_{\varepsilon}+\eta_{\varepsilon}+\varepsilon Q\left(u_{\varepsilon}\right)=L \quad\left(\text { in } X^{*}\right) .
$$

The set $\left\{u_{\varepsilon}: \varepsilon \in(0,1)\right\}$ is a subset of $\overline{B_{R}(0)}$ and is thus bounded in $X$. From the boundedness of $B$ and $Q$, we see that $\left\{\eta_{\varepsilon}: \varepsilon \in(0,1)\right\}$ and $\left\{Q\left(u_{\varepsilon}\right): \varepsilon \in(0,1)\right\}$ are bounded subsets of $X^{*}$. As a consequence of (2.26), the set $\left\{\zeta_{\varepsilon}: \varepsilon \in(0,1)\right\}$ is also bounded in $X^{*}$.

In view of the reflexivity of $X$, there exist a sequence $\left\{\varepsilon_{n}\right\} \subset(0,1), \varepsilon_{n} \rightarrow 0^{+}$(as $n \rightarrow \infty)$ and $u \in X, \zeta, \eta \in X^{*}$ such that

$$
\begin{gathered}
u_{\varepsilon_{n}} \rightarrow u \text { (weakly) in } X, \\
\zeta_{\varepsilon_{n}} \rightarrow^{*} \zeta, \eta_{\varepsilon_{n}} \rightarrow^{*} \eta\left(\text { weak }^{*}\right) \text { in } X^{*} .
\end{gathered}
$$

The rest of the proof in this step follows the same lines as those in Theorem 5.2 and Corollary 5.2 in 10 and is presented here for the sake of completeness. Since $\left\{\left\langle\zeta_{\varepsilon_{n}}, u_{\varepsilon_{n}}-u\right\rangle\right\}$ and $\left\{\left\langle\eta_{\varepsilon_{n}}, u_{\varepsilon_{n}}-u\right\rangle\right\}$ are bounded sequences in $\mathbb{R}$, by passing to subsequences if necessary, we can assume that

$$
\left\langle\zeta_{\varepsilon_{n}}, u_{\varepsilon_{n}}-u\right\rangle \rightarrow \chi_{1},\left\langle\eta_{\varepsilon_{n}}, u_{\varepsilon_{n}}-u\right\rangle \rightarrow \chi_{2} \text { as } n \rightarrow \infty .
$$

As $\varepsilon_{n} Q\left(u_{\varepsilon_{n}}\right) \rightarrow 0$ in $X^{*}$ and $\left\langle L, u_{\varepsilon_{n}}-u\right\rangle \rightarrow 0$, it follows from (2.26) that

$$
\chi_{1}+\chi_{2}=0,
$$

and thus either $\chi_{1} \leq 0$ or $\chi_{2} \leq 0$. Assume first that $\chi_{1} \leq 0$. We see from (2.29) that $\lim \sup \left\langle\eta_{\varepsilon_{n}}, u_{\varepsilon_{n}}\right\rangle \leq \lim \left\langle\eta_{\varepsilon_{n}}, u\right\rangle=\langle\eta, u\rangle$. Since $B$ is pseudomonotone, this limit together with (2.27) and (2.28) implies that

$$
\eta \in B(u),
$$

and $\lim \left\langle\eta_{\varepsilon_{n}}, u_{\varepsilon_{n}}\right\rangle=\langle\eta, u\rangle$, i.e., $\chi_{1}=0$, and thus $\chi_{2}=0$, which in view of (2.29) means that

$$
\lim \left\langle\zeta_{\varepsilon_{n}}, u_{\varepsilon_{n}}\right\rangle=\lim \left\langle\zeta_{\varepsilon_{n}}, u\right\rangle=\langle\zeta, u\rangle .
$$

Invoking Proposition 2.2(b) of [10, we see that this limit, together with (2.27)(2.28) and the maximal monotonicity of $A+\partial I_{R}$, implies that

$$
u \in D\left(A+\partial I_{R}\right) \text { and } \zeta \in\left(A+\partial I_{R}\right)(u) .
$$

It follows from (2.26) that

$$
\zeta_{\varepsilon_{n}}+\eta_{\varepsilon_{n}}+\varepsilon_{n} Q\left(u_{\varepsilon_{n}}\right)=L, \forall n .
$$

By passing to the weak* limit in (2.33) and taking into account (2.28), (2.31), and (2.32), we see that $u$ is a solution of the inclusion

$$
\left(A+\partial I_{R}\right)(u)+B(u) \ni L .
$$

Now assume that $\chi_{2} \leq 0$. We have $\lim \sup \left\langle\zeta_{\varepsilon_{n}}, u_{\varepsilon_{n}}\right\rangle \leq \lim \left\langle\zeta_{\varepsilon_{n}}, u\right\rangle=\langle\zeta, u\rangle$. Using again Proposition 2.2(b) in 10, we obtain from this limit, (2.27), (2.28), and the maximal monotonicity of $A+\partial I_{R}$ that (2.32) holds in this case as well. Furthermore, $\lim \left\langle\zeta_{\varepsilon_{n}}, u_{\varepsilon_{n}}\right\rangle=\langle\zeta, u\rangle$. This shows that $\chi_{2}=0$ and thus $\chi_{1}=0$ thanks to (2.30). From the above arguments, we have again (2.33) and $u$ is a solution of (2.34). 
Step 6. We have shown from Step 1 to Step 5 that for each $R>0$ such that $D(A) \cap B_{R}(0) \neq \emptyset$, the inclusion (2.34) has a solution $u=u_{R} \in D\left(A+\partial I_{R}\right)(=$ $\left.D(A) \cap \overline{B_{R}(0)}\right)$; that is, there are $u_{R} \in D(A) \cap \overline{B_{R}(0)}, \zeta_{R} \in\left(A+\partial I_{R}\right)\left(u_{R}\right)$, and $\eta_{R} \in B\left(u_{R}\right)$ such that $\zeta_{R}+\eta_{R}=L$. Moreover, $\zeta_{R}=\xi_{R}+l_{R}$ with $\xi_{R} \in A\left(u_{R}\right)$ and $l_{R} \in \partial I_{R}\left(u_{R}\right)$. The above equation gives

$$
l_{R}=L-\xi_{R}-\eta_{R},
$$

which implies that

$$
I_{R}(v)-I_{R}\left(u_{R}\right) \geq\left\langle l_{R}, v-u_{R}\right\rangle=\left\langle L-\xi_{R}-\eta_{R}, v-u_{R}\right\rangle, \forall v \in X .
$$

Since $I_{R}\left(u_{R}\right)=0$, we have

$$
0 \geq\left\langle L-\xi_{R}-\eta_{R}, v-u_{R}\right\rangle, \forall v \in \overline{B_{R}(0)} .
$$

Let $u_{0} \in X$ be as in the assumption of Theorem 2.2. For all $R$ such that $R \geq\left\|u_{0}\right\|$ and $B_{R}(0) \cap D(A) \neq \emptyset$, letting $v=u_{0}$ in this inequality yields

$$
\left\langle\xi_{R}+\eta_{R}-L, u_{R}-u_{0}\right\rangle \leq 0 .
$$

Then, for $R$ satisfying (2.1), since $u_{R} \in D(A)$, we must have $\left\|u_{R}\right\| \neq R$ and thus $u_{R} \in B_{R}(0)$. This implies that $\partial I_{R}\left(u_{R}\right)=\{0\}$ and hence $l_{R}=0$, which, together with (2.35), shows that $u=u_{R}$ satisfies the inclusion (2.2) and completes our proof.

In the next result, we present several sufficient conditions for the coercivity condition (2.1), some of which appeared in various existence and range theorems (cf. e.g. [1, 2, 9, 10, 11, 12, 14, etc.).

Corollary 2.3. Let $A$ and $B$ be as in Theorem 2.2. Under one of the following sufficient conditions for (2.1), the inclusion (2.2) has a solution, that is, $L \in$ $R(A+B)$.

(a) There exist $u_{0} \in X$ and $R \geq\left\|u_{0}\right\|$ such that $D(A) \cap B_{R}(0) \neq \emptyset$ and

$$
\inf _{u \in D(A) \cap S_{R}(0), \xi \in A(u), \eta \in B(u)}\left\langle\xi+\eta-L, u-u_{0}\right\rangle>0,
$$

(b) $\exists u_{0} \in X: \liminf _{\|u\| \rightarrow \infty, u \in D(A)}\left(\inf _{\xi \in A(u), \eta \in B(u)}\left\langle\xi+\eta-L, u-u_{0}\right\rangle\right)>0$,

(c) $\exists u_{0} \in X: \limsup _{R \rightarrow \infty}\left(\inf _{u \in D(A),\|u\|=R, \xi \in A(u), \eta \in B(u)}\left\langle\xi+\eta-L, u-u_{0}\right\rangle\right)>0$,

(d) $\exists u_{0} \in X: \lim _{\|u\| \rightarrow \infty, u \in D(A)}\left(\inf _{\xi \in A(u), \eta \in B(u)}\left\langle\xi+\eta-L, u-u_{0}\right\rangle\right)=\infty$,

(e) $\exists u_{0} \in X: \liminf _{\|u\| \rightarrow \infty, u \in D(A)}\left(\inf _{\xi \in A(u), \eta \in B(u)} \frac{\left\langle\xi+\eta, u-u_{0}\right\rangle}{\|u\|}\right)>\|L\|_{*}$,

(f) $\exists u_{0} \in X: \liminf _{\|u\| \rightarrow \infty, u \in D(A)}\left(\inf _{\xi \in A(u), \eta \in B(u)} \frac{\left\langle\xi+\eta, u-u_{0}\right\rangle}{\|u\|}\right)=\infty$,

(g) $\exists u_{0} \in X: \limsup _{R \rightarrow \infty}\left(\inf _{u \in D(A),\|u\|=R, \xi \in A(u), \eta \in B(u)} \frac{\left\langle\xi+\eta, u-u_{0}\right\rangle}{R}\right)>\|L\|_{*}$,

(h) $\exists u_{0} \in X: \lim _{\|u\| \rightarrow \infty, u \in D(A)}\left(\inf _{\xi \in A(u), \eta \in B(u)} \frac{\left\langle\xi+\eta, u-u_{0}\right\rangle}{\|u\|}\right)=\infty$.

Remark 2.4. (a) The existence of a function similar to $f$ in Step 1 in the proof of Theorem 2.2 has been shown for quasibounded operators in Lemma 2.1 of [7]. 
(b) Theorem 2.2 above does not imply Theorem 2.1, 7], since the latter is about quasibounded, densely defined generalized pseudomonotone operators. On the other hand, Theorem 2.1, 7], does not contain Theorem 2.2 here since condition (2.1) above is on a sphere, which could be small or large, rather than on a region outside a ball as in condition $(*)$ in Theorem 2.1, 7]. Furthermore, condition (2.1) is on $L$ only for it to belong to the range of $A+B$ rather than on a set $S$ to obtain $S \subset \overline{R(A+B)}$ and $\operatorname{int} S \subset \operatorname{int} R(A+B)$ as in the conclusion of Theorem 2.1, [7.

Based on a new degree theory for sums of maximal monotone and densely defined operators of class $\left(\tilde{S}_{+}\right)$, conditions were given in Theorem 6.1 of $[8$ on the sum $T+C$ for the existence of zeros of that sum which are very natural and verifiable (see also Theorems 6.2 and 5.8 in [8]). One of the conditions is a Leray-Schauder condition, and the other is its inner product counterpart, which is similar to condition (2.1) above. Theorem 6.1 in [8] and Theorem 2.2 here are again not contained in each other since the operator $T$ in Theorem 6.1 of $[8$ is monotone with domain containing a dense subspace and $C$ is a single-valued generalized pseudomonotone operator. As is seen later, one of our main interests here is in variational inequalities in which the maximal monotone operators are given by subdifferentials of convex functionals whose effective domains are usually not dense subsets of $X$.

(c) Condition (h) is the most restrictive among those in Corollary 2.3 In many particular cases of single-valued mappings and/or without one of the two components $\xi$ and $\eta$, it is usually referred to as a coercivity condition.

(d) Corollary 2.3 with condition (h) improves and unifies both Theorem 5.2 and Corollary 5.2 of [10] in several ways. For example, as seen below, this allows us to include the case of variational inequalities as a particular case of Corollary 2.3 or Theorem 2.2. Note that the definition of pseudomonotone mappings in [10] is in fact that of everywhere defined generalized pseudomonotone mappings (cf. 44). However, due to the boundedness assumption, the two definitions are equivalent.

Also from the Corollary 2.3(h), we see that condition (a) in Corollary 5.2, 10, can be completely eliminated. This elimination of that condition in Corollary 5.2 of [10] is particularly relevant in applications to partial differential equations, since the operators in such equations or related variational inequalities do not generally have a linear growth from below. Furthermore, $u_{0}$ is not assumed to be in the domain $D(A)$ of $A$ (see Theorem 5.2 in [10]).

Note that Corollary 5.2 in 10 does not imply Theorem 5.2 there. However, with the improvement made above (by eliminating Condition (a) in Corollary 5.2, 10), Corollary 2.3(h) (and thus Theorem 2.2) here implies Theorem 5.2 in [10. In fact, suppose the hypothesis of Theorem 5.2 in 10 is fulfilled; that is, suppose there exists $u_{0} \in D(A)$ such that

$$
\lim _{\|u\| \rightarrow \infty, u \in D(A)}\left(\frac{\left\langle\eta, u-u_{0}\right\rangle}{\|u\|}\right)=\infty .
$$

Let $\xi_{0}$ be a fixed element of $A\left(u_{0}\right)$. We have for any $\xi \in A(u)$,

$$
\begin{aligned}
\left\langle\xi+\eta, u-u_{0}\right\rangle & =\left\langle\xi-\xi_{0}, u-u_{0}\right\rangle+\left\langle\eta, u-u_{0}\right\rangle+\left\langle\xi_{0}, u-u_{0}\right\rangle \\
& \geq\left\langle\eta, u-u_{0}\right\rangle+\left\langle\xi_{0}, u-u_{0}\right\rangle .
\end{aligned}
$$

Hence,

$$
\frac{\left\langle\xi+\eta, u-u_{0}\right\rangle}{\|u\|} \geq \frac{\left\langle\eta, u-u_{0}\right\rangle}{\|u\|}-\left\|\xi_{0}\right\|_{*}\left(1+\frac{\left\|u_{0}\right\|}{\|u\|}\right),
$$


which means that (2.37) implies condition (h) of Corollary 2.3] i.e., Corollary 2.3 (h) generalizes Theorem 5.2, 10] and also Theorem 3.2, 9.

(e) The boundedness of $B$ can be somewhat relaxed to other boundedness assumptions such as (pm4) in [9].

We consider now some further corollaries of the above results related to variational inequalities. Assume $K \neq \emptyset$ is a closed, convex subset of $X$ and $\psi$ : $X \rightarrow \mathbb{R} \cup\{\infty\}$ is a proper, convex, and lower semicontinuous functional such that $K \cap D(\psi) \neq \emptyset$. It follows that $\phi=\psi+I_{K}: X \rightarrow \mathbb{R} \cup\{\infty\}$ is also a proper, convex, and lower semicontinuous functional with $D(\phi)=D(\psi) \cap K$ and thus $\partial \phi$ is a maximal monotone operator from $X$ into $2^{X^{*}}$. Note that when $K=X$, we have $\phi=\psi$. Applying Theorem 2.2 to the case where $A=\partial \phi$ yields the following corollary.

Corollary 2.5. Let $L \in X^{*}$ and assume there exist $u_{0} \in X$ and $R>\left\|u_{0}\right\|$ such that

$$
D(\partial \phi) \cap B_{R}(0) \neq \emptyset
$$

and

$$
\left\langle\xi+\eta-L, u-u_{0}\right\rangle>0,
$$

for all $u \in D(\partial \phi)$ with $\|u\|=R$, all $\xi \in \partial \phi(u)$, all $\eta \in B(u)$. Then there exist $u \in D(\partial \phi)(\subset D(\phi)=K \cap D(\psi))$ and $\eta \in B(u)$ that satisfy the variational inequality

$$
\langle\eta-L, v-u\rangle+\psi(v)-\psi(u) \geq 0, \forall v \in K .
$$

Condition (2.38) has the following particular case, which is closer to the coercivity conditions usually appearing in existence theorems for variational inequalities.

Corollary 2.6. Let $L \in X^{*}$ and suppose there exist $u_{0} \in D(\psi) \cap K$ and $R>\left\|u_{0}\right\|$ such that

$$
D\left(\partial\left(\psi+I_{K}\right)\right) \cap B_{R}(0) \neq \emptyset
$$

and

$$
\left\langle\eta-L, u-u_{0}\right\rangle+\psi(u)>\psi\left(u_{0}\right),
$$

for all $u \in D(\psi) \cap K$ with $\|u\|=R$, all $\eta \in B(u)$. Then the variational inequality (2.39) has solutions.

We note as above that condition (2.40) has in its turn several sufficient conditions such as:

$$
\begin{gathered}
\liminf _{\|u\| \rightarrow \infty, u \in D(\psi) \cap K, \eta \in B(u)}\left[\left\langle\eta-L, u-u_{0}\right\rangle+\psi(u)\right]>\psi\left(u_{0}\right), \\
\liminf _{\|u\| \rightarrow \infty, u \in D(\psi) \cap K, \eta \in B(u)}\left[\left\langle\eta-L, u-u_{0}\right\rangle+\psi(u)\right]=\infty, \\
\liminf _{\|u\| \rightarrow \infty, u \in D(\psi) \cap K, \eta \in B(u)} \frac{\left\langle\eta, u-u_{0}\right\rangle+\psi(u)}{\|u\|}>\|L\|_{*},
\end{gathered}
$$

or, more restrictively,

$$
\liminf _{\|u\| \rightarrow \infty, u \in D(\psi) \cap K, \eta \in B(u)} \frac{\left\langle\eta, u-u_{0}\right\rangle+\psi(u)}{\|u\|}=\infty .
$$

Under one of the above conditions from (2.41) to (2.44), the variational inequality (2.39) has solutions. Note that condition (2.44) is usually referred to as a coercivity condition for (2.39). 
Remark 2.7. (a) In the particular case where $K=X$ and condition (2.44) is taken into account, we obtain Corollary 5.3 in [10] without the extra growth condition assumed there.

(b) Corollaries 2.5 and 2.6 show that we can derive existence theorems for variational inequalities containing multivalued pseudomonotone operators as direct consequences of our existence results for pseudomonotone perturbations of maximal monotone operators without following a parallel, independent path as in Section 4 of 9 .

\section{An EXAmple}

Let us conclude our discussions with a simple example to illustrate the above abstract theorems. The example is on a variational inequality in a Sobolev space with variable exponent. Let $\Omega$ be a bounded region in $\mathbb{R}^{N}(N \geq 1)$ with sufficiently smooth boundary $\partial \Omega$. Let $p$ be a function in $C(\bar{\Omega})$ such that $p_{-}=\min \{p(x): x \in$ $\bar{\Omega}\}>1$ and let $L^{p(\cdot)}(\Omega)$ and $W^{1, p(\cdot)}(\Omega)$ be respectively the Lebesgue and first order Sobolev spaces with variable exponent $p(\cdot)$. The norms on $L^{p(\cdot)}(\Omega)$ and $W^{1, p(\cdot)}(\Omega)$ are given by

$$
\|u\|_{L^{p(\cdot)}(\Omega)}=\inf \left\{k>0: \int_{\Omega}\left|\frac{u(x)}{k}\right|^{p(x)} d x \leq 1\right\}\left(u \in L^{p(\cdot)}(\Omega)\right)
$$

and

$$
\|u\|=\|u\|_{W^{1, p(\cdot)}(\Omega)}=\|u\|_{L^{p(\cdot)}(\Omega)}+\|\nabla u\|_{L^{p(\cdot)}(\Omega)}\left(u \in W^{1, p(\cdot)}(\Omega)\right) .
$$

$L^{p(\cdot)}(\Omega)$ and thus $W^{1, p(\cdot)}(\Omega)$ are separable and reflexive Banach spaces. Let $\left[W^{1, p(\cdot)}(\Omega)\right]^{*}$ denote the dual of and $\langle\cdot, \cdot\rangle$ the dual pairing between $W^{1, p(\cdot)}(\Omega)$ and $\left[W^{1, p(\cdot)}(\Omega)\right]^{*}$. Assume $\Gamma$ is a measurable subset of $\partial \Omega$ with positive (surface) measure and let

$$
W_{\Gamma}^{1, p(\cdot)}(\Omega)=\left\{u \in W^{1, p(\cdot)}(\Omega):\left.u\right|_{\partial \Omega}(x)=0 \text { for a.e. } x \in \Gamma\right\},
$$

where $\left.u\right|_{\partial \Omega}$ is the trace of $u$ on $\partial \Omega$ (it is known that $\left.u\right|_{\partial \Omega} \in L^{p(\cdot)}(\partial \Omega)$; cf. [5]). $W_{\Gamma}^{1, p(\cdot)}(\Omega)$ is also a separable reflexive Banach space with the norm $\|\cdot\|$ restricted to $W_{\Gamma}^{1, p(\cdot)}(\Omega)$. We shall be able to define an equivalent norm on $W_{\Gamma}^{1, p(\cdot)}(\Omega)$ thanks to the following Poincaré inequality for that space.

Lemma 3.1. There exists $C_{0}>0$ such that

$$
\|u\|_{L^{p(\cdot)}(\Omega)} \leq C_{0}\||\nabla u|\|_{L^{p(\cdot)}(\Omega)}, \forall u \in W_{\Gamma}^{1, p(\cdot)}(\Omega) .
$$

Inequality (3.1) can be proved by following the same lines as in the proof of the regular Poincaré inequality in Sobolev spaces with variable exponents (i.e. when $\Gamma=$ $\partial \Omega$ ) presented in Theorem 7 of [6]. We just notice that by using the bootstrapping argument in that theorem, we can reduce the proof of (3.1) to the corresponding inequality in $L^{1}(\Omega)$ and $W_{\Gamma}^{1,1}(\Omega)=\left\{u \in W^{1,1}(\Omega):\left.u\right|_{\partial \Omega}=0\right.$ a.e. on $\left.\Gamma\right\}$, which is a classical result.

Let $h \in W^{1, p(\cdot)}(\Omega)$ and let $K$ be a closed convex subset of the linear manifold

$$
\begin{aligned}
h+W_{\Gamma}^{1, p(\cdot)}(\Omega) & =\left\{h+w: w \in W_{\Gamma}^{1, p(\cdot)}(\Omega)\right\} \\
& =\left\{u \in W^{1, p(\cdot)}(\Omega): u(x)=h(x) \text { for a.e. } x \in \Gamma\right\} .
\end{aligned}
$$


Assume $A: \Omega \times \mathbb{R}^{N} \rightarrow \mathbb{R}$ is a Carathéodory function that satisfies the following conditions:

$$
|A(x, \xi)| \leq a_{1}(x)+b_{1}|\xi|^{p(x)-1}, \text { for a.e. } x \in \Omega \text {, all } \xi \in \mathbb{R}^{N},
$$

with $a_{1} \in L^{p^{\prime}(\cdot)}(\Omega)\left(p^{\prime}(\cdot)\right.$ is the Hölder conjugate of $\left.p(\cdot)\right)$ and $b_{1}>0$,

$$
\left[A(x, \xi)-A\left(x, \xi^{\prime}\right)\right] \cdot\left(\xi-\xi^{\prime}\right) \geq 0, \text { for a.e. } x \in \Omega \text {, all } \xi, \xi^{\prime} \in \mathbb{R}^{N},
$$

and there are $a_{2} \in L^{1}(\Omega)$ and $b_{2}>0$ such that

$$
A(x, \xi) \cdot \xi \geq b_{2}|\xi|^{p(x)}-a_{2}(x), \text { for a.e. } x \in \Omega \text {, all } \xi \in \mathbb{R}^{N} \text {. }
$$

Also, we assume that $L \in\left[W^{1, p(\cdot)}(\Omega)\right]^{*}$ and $f: \Omega \times \mathbb{R} \rightarrow \mathbb{R}$ is a Carathéodory function. We are interested here in the variational inequality:

$$
\left\{\begin{array}{l}
\int_{\Omega} A(x, \nabla u) \cdot(\nabla v-\nabla u) d x+\int_{\Omega} f(x, u)(v-u) d x \geq\langle L, v-u\rangle, \forall v \in K \\
u \in K
\end{array}\right.
$$

Let us prove the following existence theorem for (3.5).

Theorem 3.2. Assume $f$ has the following growth condition:

$$
|f(x, u)| \leq a_{1}(x)+b_{1}|u|^{p^{*}(x)-1},
$$

for a.e. $x \in \Omega$, all $u \in \mathbb{R}$, where $b_{1} \geq 0$ and $a_{1} \in L^{\left(p^{*}\right)^{\prime}(\cdot)}\left(p^{*}(\cdot)\right.$ is the Sobolev conjugate of $p(\cdot)$ and $\left(p^{*}\right)^{\prime}(\cdot)$ is the Hölder conjugate of $\left.p^{*}(\cdot)\right)$. Moreover,

$$
f=g+h,
$$

where $g, h: \Omega \times \mathbb{R} \rightarrow \mathbb{R}$ are Carathéodory functions such that

$$
g(x, \cdot) \text { is nondecreasing from } \mathbb{R} \text { to } \mathbb{R},
$$

for a.e. $x \in \Omega$, and $h$ has a "sublinear" growth:

$$
|h(x, u)| \leq a_{2}(x)+b_{2}|u|^{q(x)-1}(\text { a.e. } x \in \Omega \text {, all } u \in \mathbb{R}),
$$

where $b_{2} \geq 0, q \in C(\bar{\Omega})$ with $1 \leq q_{-} \leq q_{+}<p_{-}$, and $a_{2} \in L^{q^{\prime}(\cdot)}$ (where $q_{-}=$ $\inf \{q(x): x \in \bar{\Omega}\}$ and $\left.q_{+}=\sup \{q(x): x \in \bar{\Omega}\}\right)$.

Then, the variational inequality (3.5) has solutions.

Proof. Let $\mathcal{A}, \mathcal{G}, \mathcal{H}, \mathcal{F}: W^{1, p(\cdot)}(\Omega) \rightarrow\left[W^{1, p(\cdot)}(\Omega)\right]^{*}$ be defined by $\langle\mathcal{A}(u), v\rangle=$ $\int_{\Omega} A(x, \nabla u) \cdot \nabla v d x,\langle\mathcal{G}(u), v\rangle=\int_{\Omega} g(x, u) v d x,\langle\mathcal{H}(u), v\rangle=\int_{\Omega} h(x, u) v d x$, and $\mathcal{F}(u)$ $=\mathcal{G}(u)+\mathcal{H}(u)$, for all $u, v \in W^{1, p(\cdot)}(\Omega)$. Then, $\mathcal{A}, \mathcal{G}, \mathcal{H}, \mathcal{F}$ are bounded and continuous on $W^{1, p(\cdot)}(\Omega)$. With the above notation, we note that 3.5 is the same as the inequality

$$
\langle(\mathcal{A}+\mathcal{F})(u)-L, v-u\rangle+I_{K}(v)-I_{K}(u) \geq 0, \forall v \in K,
$$

which is equivalent to the inclusion

$$
\left(\mathcal{A}+\mathcal{F}+\partial I_{K}\right)(u) \ni L .
$$

It follows from (3.3) and $(3.8)$ that $\mathcal{A}$ and $\mathcal{G}$ are monotone bounded operators on $W^{1, p(\cdot)}(\Omega)$ and from 3.9 that $\mathcal{H}$ is completely continuous there. Thus $\mathcal{A}+\mathcal{F}$ is pseudomonotone and bounded on $W^{1, p(\cdot)}(\Omega)$. Since $\partial I_{K}$ is maximal monotone on 
that space, to apply the above existence theorem, we just need to check one of the above coercivity conditions, namely, the following condition:

$$
\left\langle(\mathcal{A}+\mathcal{F})(u)-L, u-u_{0}\right\rangle+I_{K}(u) \rightarrow \infty \text { as }\|u\| \rightarrow \infty,
$$

or equivalently,

$$
\left\langle(\mathcal{A}+\mathcal{F})(u)-L, u-u_{0}\right\rangle \rightarrow \infty \text { as }\|u\| \rightarrow \infty, u \in K,
$$

for some (fixed) $u_{0} \in K$. In fact, let $u_{0}$ be any (fixed) element of $K$. As a consequence of (3.1), there exists $C_{1}>0$ such that $\||\nabla u|\|_{L^{p(\cdot)}(\Omega)} \geq\|u\|$ for all $u \in W_{\Gamma}^{1, p(\cdot)}(\Omega)$. Let $u \in K$. Since $u-u_{0} \in W_{\Gamma}^{1, p(\cdot)}(\Omega)$, we have

$$
\begin{aligned}
\||\nabla u|\|_{L^{p(\cdot)}(\Omega)} & \geq\left\|\left|\nabla\left(u-u_{0}\right)\right|-\left|\nabla u_{0}\right|\right\|_{L^{p(\cdot)}(\Omega)} \\
& \geq C_{1}\left\|u-u_{0}\right\|-\left\|\left|\nabla u_{0}\right|\right\|_{L^{p(\cdot)}(\Omega)} \\
& \geq C_{1}\|u\|-C_{2},
\end{aligned}
$$

where $C_{2}$ is a positive constant independent of $u \in K$. Combining (3.13) with (3.4), we see that

$$
\int_{\Omega} A(x, \nabla u) \cdot \nabla u d x \geq b_{2} \int_{\Omega}|\nabla u|^{p(x)} d x-\left\|a_{2}\right\|_{L^{1}(\Omega)} .
$$

Moreover, if $u \in K$ and $\|u\|$ is sufficiently large, then $\||\nabla u|\|_{L^{p(\cdot)}(\Omega)}>1$ and

$$
\int_{\Omega}|\nabla u|^{p(x)} d x \geq\||\nabla u|\|_{L^{p(\cdot)}(\Omega)}^{p_{-}} .
$$

By (3.2) and Young's inequality (with $\varepsilon$ ), we have

$$
\begin{aligned}
\left|\int_{\Omega} A(x, \nabla u) \cdot \nabla u_{0} d x\right| \leq & \int_{\Omega} a_{1}\left|\nabla u_{0}\right| d x+b_{1} \int_{\Omega}|\nabla u|^{p(x)-1}\left|\nabla u_{0}\right| d x \\
\leq & \left(\frac{1}{p-}+\frac{1}{p_{-}^{\prime}}\right)\left\|a_{1}\right\|_{L^{p^{\prime}(\cdot)}(\Omega)}\left\|\left|\nabla u_{0}\right|\right\|_{L^{p(\cdot)}(\Omega)} \\
& +\varepsilon \int_{\Omega}|\nabla u|^{p(x)} d x+C_{3}(\varepsilon) \int_{\Omega}\left|\nabla u_{0}\right|^{p(x)} d x
\end{aligned}
$$

where $\varepsilon>0$ and $C_{3}(\varepsilon)>0$ depends on $\varepsilon$ (and other constants, but not on $u$ ). On the other hand, since $g(x, \cdot)$ is nondecreasing and $g\left(\cdot, u_{0}\right)$ belongs to $L^{\left(p^{*}\right)^{\prime}(\cdot)}(\Omega)$, we have

$$
\begin{aligned}
\left\langle\mathcal{G}(u), u-u_{0}\right\rangle & \geq \int_{\Omega} g\left(x, u_{0}\right)\left(u-u_{0}\right) d x \\
& \geq-\left\|g\left(\cdot, u_{0}\right)\right\|_{L^{\left(p^{*}\right)^{\prime}(\cdot)}(\Omega)}\left(\|u\|_{L^{p^{*}(\cdot)}(\Omega)}+\left\|u_{0}\right\|_{L^{p^{*}(\cdot)}(\Omega)}\right) \\
& \geq-C_{4}\|u\|-C_{5} .
\end{aligned}
$$

Also, it follows from (3.9) that

$$
\begin{aligned}
\left|\left\langle\mathcal{H}(u), u-u_{0}\right\rangle\right| \leq & \int_{\Omega}\left(a_{2}+b_{2}|u|^{q(x)-1}\right)\left(|u|+\left|u_{0}\right|\right) d x \\
\leq & \left\|a_{2}\right\|_{L^{q^{\prime}(\cdot)}(\Omega)}\left\|u_{0}\right\|_{L^{q(\cdot)}(\Omega)}+\left\|a_{2}\right\|_{L^{q^{\prime}(\cdot)}(\Omega)}\|u\|_{L^{q(\cdot)}(\Omega)} \\
& +b_{2}\left(\|u\|_{L^{q(\cdot)}(\Omega)}^{q+1)}\right. \\
\leq & C_{6}+C_{7}\|u\|^{q+}+C_{8}\|u\| .
\end{aligned}
$$

Lastly, we have

$$
\left|\left\langle L, u-u_{0}\right\rangle\right| \leq\|L\|_{\left[W^{1, p(\cdot)}(\Omega)\right]^{*}}\left(\|u\|+\left\|u_{0}\right\|\right) .
$$


Combining (3.14) with (3.16) (3.19) and $\varepsilon=b_{2} / 2$ in (3.16), we obtain, for all $u \in K$ with $\|u\|$ sufficiently large,

$$
\left\langle\mathcal{A}+\mathcal{F}-L, u-u_{0}\right\rangle \geq \frac{b_{2}}{2} \int_{\Omega}|\nabla u|^{p(x)} d x-C_{7}\|u\|^{q_{+}}-C_{9}\|u\|-C_{10} .
$$

From (3.15) and (3.13), we see that for such a function $u$,

$$
\int_{\Omega}|\nabla u|^{p(x)} d x \geq C_{1}^{p_{-}}\|u\|^{p_{-}}-C_{11}
$$

and thus,

$$
\left\langle\mathcal{A}+\mathcal{F}-L, u-u_{0}\right\rangle \geq C_{1}^{p_{-}}\|u\|^{p_{-}}-C_{7}\|u\|^{q_{+}}-C_{9}\|u\|-C_{12} .
$$

Since $p_{-}>1$ and $p_{-}>q_{+}$, this estimate implies (3.12) (i.e. (3.11)) and our proof is complete.

Remark 3.3. Note that without the maximal monotone operator $\partial I_{K}$, the pseudomonotone operator $\mathcal{A}+\mathcal{F}-L$ is not coercive on $W^{1, p(\cdot)}(\Omega)$; that is, it does not satisfy the coercivity condition

$$
\lim _{\|u\| \rightarrow \infty} \frac{\left\langle(\mathcal{A}+\mathcal{F}-L)(u), u-u_{0}\right\rangle}{\|u\|}=\infty
$$

as usually assumed in existence theorems for pseudomonotone and maximal monotone operators (cf. e.g. 9, 10, 4, 13, etc.).

\section{ACKNOWLEDGMENT}

The author would like to thank the referee for valuable comments and additional references.

\section{REFERENCES}

[1] H. Brézis, Equations et inéquations non linéaires dans les espaces vectoriels en dualité, Ann. Inst. Fourier 18 (1968), 115-175. MR0270222 (42:5113)

[2] H. Brézis and L. Nirenberg, Characterizations of the ranges of some nonlinear operators and applications to boundary value problems, Ann. Scuola Norm. Sup. Pisa 5 (1978), 225-326. MR0513090 (58:23813)

[3] F. E. Browder, Nonlinear operators and nonlinear equations of evolution in Banach spaces, Nonlinear functional analysis (Proc. Sympos. Pure Math., Vol. XVIII, Part 2, Chicago, Ill., 1968), Amer. Math. Soc., Providence, R. I., 1976, pp. 1-308. MR0405188 (53:8982)

[4] F. E. Browder and P. Hess, Nonlinear mappings of monotone type in Banach spaces, J. Functional Analysis 11 (1972), 251-294. MR0365242 (51:1495)

[5] Xianling Fan, Boundary trace embedding theorems for variable exponent Sobolev spaces, J. Math. Anal. Appl. 339 (2008), 1395-1412. MR2377096 (2008k:46095)

[6] Xianling Fan and Dun Zhao, On the spaces $L^{p(x)}(\Omega)$ and $W^{m, p(x)}(\Omega)$, J. Math. Anal. Appl. 263 (2001), 424-446. MR.1866056(2003a:46051)

[7] Z. Guan, A. G. Kartsatos, and I. V. Skrypnik, Ranges of densely defined generalized pseudomonotone perturbations of maximal monotone operators, J. Differential Equations $\mathbf{1 8 8}$ (2003), 332-351. MR1954518 (2003m:47096)

[8] A. G. Kartsatos and I. V. Skrypnik, A new topological degree theory for densely defined quasibounded $\left(\tilde{S}_{+}\right)$-perturbations of multivalued maximal monotone operators in reflexive Banach spaces, Abstr. Appl. Anal. 2005, no. 2, 121-158. MR2179439(2006h:47075)

[9] N. Kenmochi, Nonlinear operators of monotone type in reflexive Banach spaces and nonlinear perturbations, Hiroshima Math. J. 4 (1974), 229-263. MR0383162 (52:4043)

[10] __ Monotonicity and compactness methods for nonlinear variational inequalities, Handbook of Differential Equations, Vol. IV, Elsevier/North-Holland, Amsterdam, 2007, pp. 203298. MR 2569333 
[11] D. Kinderlehrer and G. Stampacchia, An introduction to variational inequalities and their applications, Academic Press, New York, 1980. MR.567696 (81g:49013)

[12] J. L. Lions, Quelques méthodes de résolution des problèmes aux limites non linéaires, Dunod, Paris, 1969. MR0259693(41:4326)

[13] Z. Naniewicz and P. D. Panagiotopoulos, Mathematical theory of hemivariational inequalities and applications, Marcel Dekker, New York, 1995. MR1304257 (96d:47067)

[14] E. Zeidler, Nonlinear functional analysis and its applications, Vol. II/B: Nonlinear monotone operators, Springer, New York, 1990. MR1033498 (91b:47002)

Department of Mathematics and Statistics, Missouri University of Science and TechNOLOGY, Rolla, Missouri 65409

E-mail address: vy@mst.edu 